\title{
Impurity Deposition on a First Mirror Surface during Hydrogen Discharges in LHD
}

\author{
Tsuyoshi AKIYAMA, Naoaki YOSHIDA ${ }^{1)}$, Masayuki TOKITANI, Suguru MASUZAKI, \\ Kazuo KAWAHATA, Shigeki OKAJIMA ${ }^{2)}$ and Kazuya NAKAYAMA ${ }^{2)}$ \\ National Institute for Fusion Science, Toki, Gifu 509-5292, Japan \\ ${ }^{1)}$ Kyushu University, 6-1 Kasuga-kouen, Kasuga, Fukuoka 816-8580, Japan \\ ${ }^{2)}$ Chubu University, Matsumoto-cho, Kasugai-shi, Aichi 487-8501, Japan
}

(Received 28 December 2012 / Accepted 26 April 2013)

\begin{abstract}
First mirrors in fusion devices have to retain the reflectivity and the degradation mechanism and dominant plasma operations that affect the reflectivity have to be understood. The reflectivity of visible laser light (635 $\mathrm{nm})$ of a corner cube mirror is almost entirely determined by the initial hydrogen glow discharges for wall conditioning before conducting a main plasma experiment in the Large Helical Devices (LHD). The hydrogen glow discharge forms a carbon deposition layer on the mirror surface, which degrades its reflectivity. A neon glow discharge and subsequent main discharges had less effect.
\end{abstract}

(C) 2013 The Japan Society of Plasma Science and Nuclear Fusion Research

Keywords: first mirror, impurity deposition, SEM, GD-OES

DOI: $10.1585 /$ pfr.8.1402092

\section{Introduction}

Some diagnostics on fusion devices such as interferometry and spectroscopy require the installation of invessel mirrors to transmit laser light and radiation from plasmas. To retain the performance and resolution of the diagnostics, the reflectivity of first mirrors, which face the plasmas, has to be maintained. However, the reflectivity often degrades owing to plasma-surface interaction, even in the present devices. Although first mirrors can be replaced frequently at present, they should have a long lifetime in future fusion reactors to realize reliable diagnostics and high reactor operation rate.

To suppress the reflectivity degradation, the physical mechanism and its relation to the surrounding environment should be understood. In addition, we have to determine the plasma and device operations that affect the reflectivity. Various plasma-surface interactions contribute to the degradation of the reflectivity: deposition of impurities (metal, carbon) on the surface [1], sputtering by ions and charge exchange particles [2], and the formation of bubbles and blisters on the surface layer [3]. These interactions depend on the plasma conditions (main discharge, glow discharge cleaning and working gas), in-vessel conditions (wall and divertor materials and the wall temperature), and mirror location. To date, vigorous studies on how to maintain the reflectivity have been performed. Mirror materials with a low sputtering yield, such as monocrystal molybdenum and rhodium, are being developed to reduce the surface roughness by sputtering [4]. Protective measures to mitigate impurity deposition are also proposed [5]. In

author'se-mail: takiyama@ms.nifs.ac.jp situ cleaning methods have been examined in fusion devices [6] or in laboratories [7]. We have to select the optimum method for maintaining the reflectivity, depending on which factors affect the mirror reflectivity most under individual installation conditions.

We have investigated operations that degrade the reflectivity of in-vessel mirrors in the Large Helical Device (LHD) by monitoring the reflectivity. This paper describes significant degradation of the reflectivity of a corner cube mirror by hydrogen discharge cleaning and impurity deposition on the surface. Section 2 describes the specifications of the corner cube mirror installed in LHD. The changes in the reflectivity during discharge cleaning and analyses of the impurity deposition are presented in Sec. 3. Section 4 summarizes this paper.

\section{Change in Reflectivity}

\subsection{Corner cube mirror in LHD}

A corner cube mirror is often used in a laser interferometer and polarimeter. It is very useful for making a double path in a plasma because the incident and reflected laser beams are parallel. A corner cube mirror having diameter of $50 \mathrm{~mm}$ was installed in LHD for a $\mathrm{CO}_{2}$ laser dispersion interferometer [8] in 2011. The installation position is near the inner side divertor region, which consists of carbon divertor plates, as shown in Fig. 1 (a). The mirror consists of three flat mirror parts made of 316 stainless steel. As shown in Fig. 1 (b), the base is welded to the inner wall of the LHD, and the mirror is fastened by a screw to the base (electrically connected to the wall). Although the distance to the plasma depends on the magnetic config- 
(a)

(b)
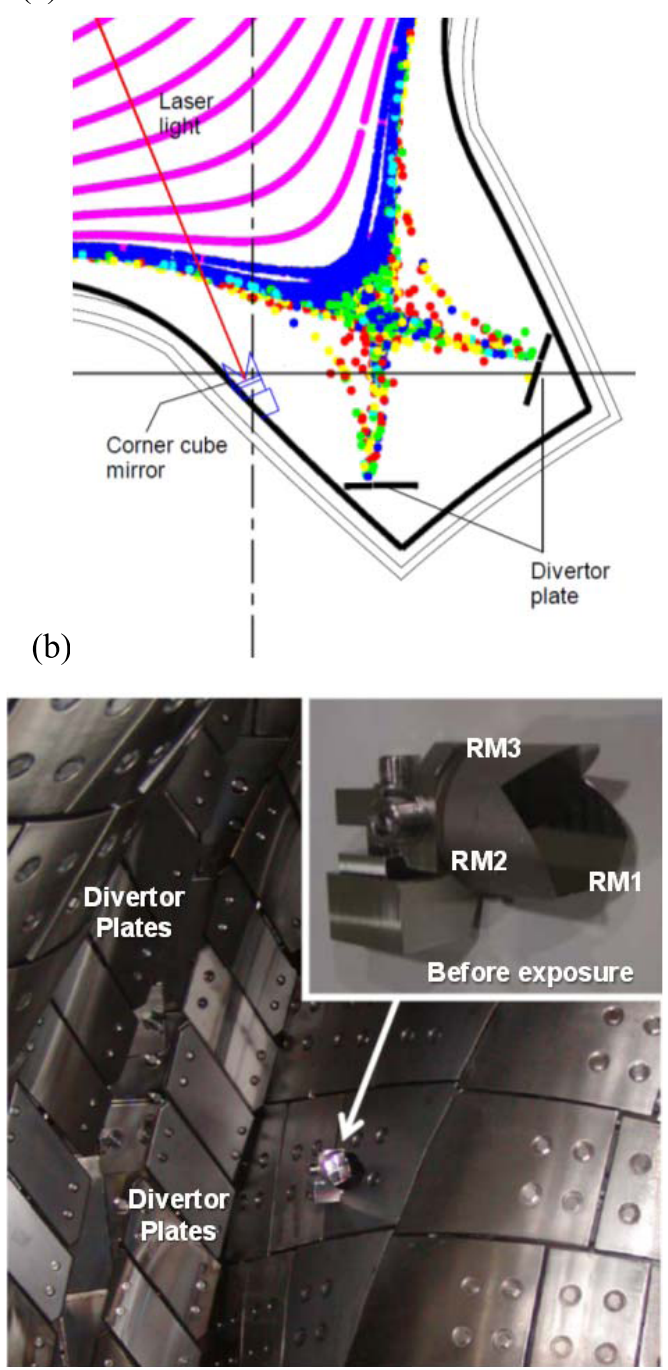

Fig. 1 (a) Position of a corner cube mirror and Poincaré plot of magnetic field lines. (b) Photograph of a corner cube mirror installed in LHD.

uration, it is roughly around $10 \mathrm{~cm}$. The mirror is usually room temperature except during baking for wall conditioning (up to around $100^{\circ} \mathrm{C}$ ). Although the mirror temperature itself was not monitored, variations in the wall temperature measured with a thermocouple close to the mirror was $\pm 2^{\circ} \mathrm{C}$ from the room temperature at most during the plasma experiments. The reflectivity before plasma exposure was $70 \%$ at $10.6 \mu \mathrm{m}\left(\mathrm{CO}_{2}\right.$ laser light $)$ for triple reflection in the reflector. Hence, the single reflection by a mirror piece was $90 \%$. The reflectivity of semiconductor laser light with a wavelength of $635 \mathrm{~nm}$ and incident power of $3 \mathrm{~mW}$ before plasma exposure was not measured. However, the reflectivity of optically polished stainless steel is usually around $60 \%$. Considering the transmissivity of the $\mathrm{ZnSe}$ window (around $60 \%$ ), the ratio of the returned beam power to the incident one should be $(0.6)^{3} \times(0.6)^{2}=0.08$ (triple reflections and double transmission for a double path in the window). This is consistent with the ratio $0.22 \mathrm{~mW} / 3 \mathrm{~mW}=0.07$ before the glow discharges begin. (a)
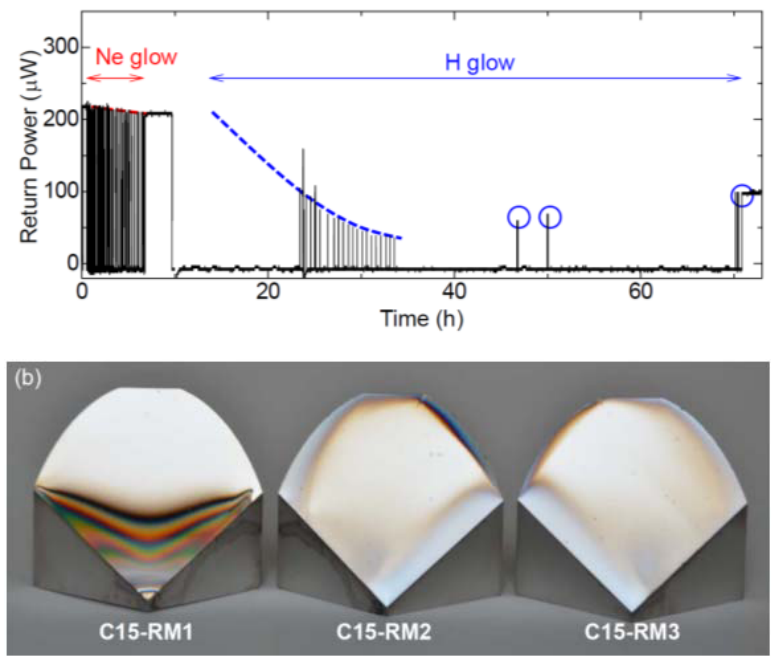

Fig. 2 (a) Temporal evolution of the power of the returned beam during $\mathrm{Ne}$ and $\mathrm{H}$ glow discharge cleanings. (b) Three mirrors making up the corner cube mirror after the 15 th experimental campaign.

\subsection{Discharge conditions}

The mirror was exposed to all glow and main discharges during the 15 th experiment campaign (51 experiment days) in 2011. The working gases were mainly hydrogen and helium for typical plasma discharges and discharge cleanings. Neon was also used for discharge cleanings before the campaign. The applied voltage was 200$300 \mathrm{~V}$ and the operational pressure was about $1 \mathrm{~Pa}$.

\subsection{Reflectivity during discharge cleaning}

Plasma-surface interactions during glow discharge cleanings and main plasma discharges are different. For example, whereas the charged particles accelerated by the sheath potential sputter the grounded material surface in a glow discharge, charge exchang neutral particles contribute to sputtering in the main discharges. Such differences cause differences in the degradation mechanism and degree of influence on the reflectivity.

Before the main plasma experiments of the 15th experiment campaign began, glow discharge cleanings with neon and hydrogen were performed for 6 and $260 \mathrm{~h}$, respectively. The power of the reflected visible laser light was monitored during the two discharges to determine how much the discharge cleanings affected the reflectivity. Since even a carbon deposition layer with a thickness of several tens of nanometers changes the color (and the reflectivity) of the mirror, the visible wavelength is preferable to monitor the surface modification. The window shutter was opened only for several tens of seconds during measurements of the power to minimize the impurity deposition on a $\mathrm{ZnSe}$ vacuum window. Figure 2 (a) shows the temporal variation in the returned beam power dur- 
ing the glow discharges. During the discharge with neon, the reflectivity degraded by only $3 \%$ during $6 \mathrm{~h}$ (roughly, $0.5 \% / \mathrm{h}$ ) despite the large energy of neon ions (strong sputtering due to the large mass). On the other hand, the reflectivity decreased by $80 \%$ over $20 \mathrm{~h}(4 \% / \mathrm{h})$ during the hydrogen glow discharge. Although the reflectivity decreased overall, it slightly increased and then decreased during the discharge. After $140 \mathrm{~h}$ (not shown), the returned power decreased to $40 \mu \mathrm{W}$. Although similar slight variations in the reflectivity were observed during the subsequence main and cleaning discharges in the experiment campaign, the final returned power was around $40 \mu \mathrm{W}$, which corresponds to a reflectivity of around $0.04 \mathrm{~mW} / 3 \mathrm{~mW} /(0.6)^{2}=4 \%$.

The reflectivity of the corner cube mirror was almost entirely determined by the above initial hydrogen glow discharge cleaning for around $20 \mathrm{~h}$.

\section{Surface Conditions and Analysis}

Figure 2 (b) shows the mirror parts after plasma exposure. An interference pattern caused by impurity deposition appears on their surfaces. The interference is significant on the surface of C15-RM1, which faces the plasma; the other two mirror parts face more toward the wall. The reflectivity varies depending on the interference pattern. On a black stripe with a width of around $1 \mathrm{~mm}$, the reflectivity for visible light decreased to around $10 \%$. The reflectivity in the yellow area between the black stripes is slightly higher, around $20 \%$. For $\mathrm{CO}_{2}$ laser light, the reflectivity is almost the same as that before plasma exposure, $90 \%$. From the number of interference stripes, the thickness of the deposition can be deduced roughly. Supposing that the refractive index $n$ of carbon is 2 , the thickness of the deposition layer should differ between neighboring stripes of the same color, $\lambda /(2 n)=160 \mathrm{~nm}$. Because there are four black stripes, the thickness of the deposition would be deduced around $600 \mathrm{~nm}$ if the deposition monotonically increases from the edge to the central region of the mirror. This is only $6 \%$ of the $\mathrm{CO}_{2}$ laser light and is too thin to change the reflectivity of this light.

Scanning electron microscopy (SEM) images and elementary analyses with energy dispersive X-ray spectrometry (EDS) at each mirror position are shown in Fig. 3. Two circular scars are due to the glow discharge optical emission spectroscopy (GD-OES) described below. At position 1 , where the surface is specular, it appears very smooth. Only the elements ( $\mathrm{Fe}, \mathrm{Cr}$, and $\mathrm{Ni}$ ) that compose the stainless steel are observed, and the impurity (carbon) deposition is less. In the area of the interference pattern (position 2), fine surface roughness with a scale of several hundreds of nano-meters is observed. The deposition of carbon, which is expected to originate in the divertor plates, is detected on the surface in addition to the elements of the stainless steel. At position 3, the central region of the corner cube mirror, the surface appears very smooth. A stronger carbon signal is detected at this point. However,
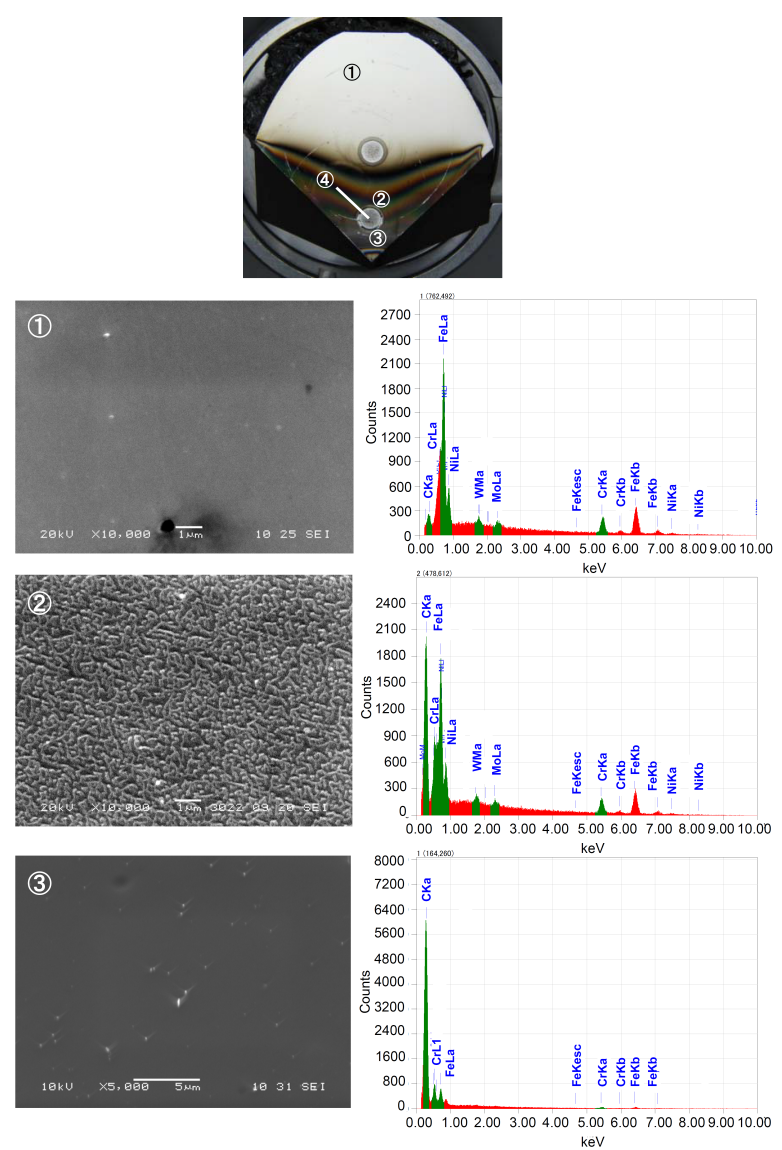

Fig. 3 SEM images and EDS spectra of the mirror surface of C15-RM1 in Fig. 1.

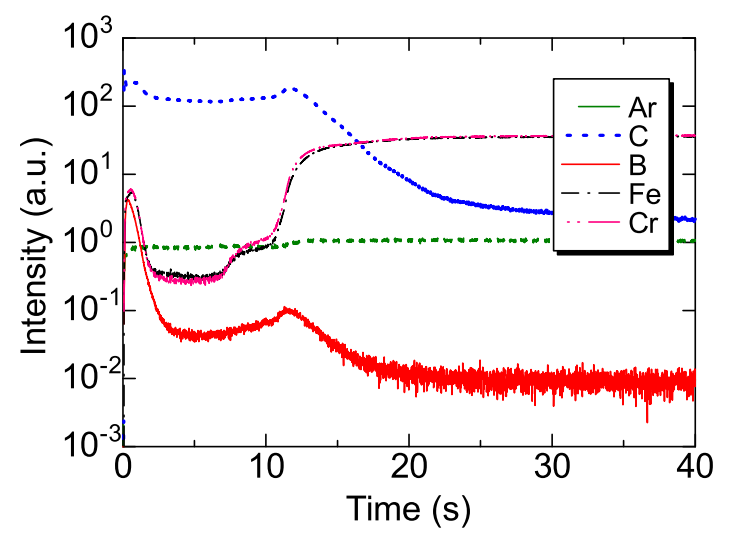

Fig. 4 Results of GD-OES analysis at position 4 of C15-RM1 in Fig. 3.

because the acceleration voltage of the electron beam is half that used in other analyses, it is difficult to compare the amount of carbon present among the analyzed positions.

The composition of the deposition layer at position 4 and the depth profiles of deposited elements were measured by GD-OES. Because GD-OES sputters the surface of a specimen by a glow discharge and determines the el- 
ement spectroscopically, the horizontal axis of Fig. 4 corresponds to the depth from the mirror surface. Similar to the EDS results, the GD-OES results indicate that carbon was deposited. The surface of the reflector appears after $t=10.5 \mathrm{~s}$. This might deposit during the first neon glow discharge, whose sputtering (particle energy) is strong. Boron is detected just at the surface of the deposition layer. As shown by the temporal evolution of Ar, which was the working gas of the glow discharge, the plasma became stable after $t=0.1 \mathrm{~s}$. The width of the boron peak is so much wider than the unstable period that it indicates the existence of boron, not a false signal due to an unstable plasma condition. Because boronization was performed just before the start of the main plasma discharge, this indicates that a thick carbon deposition layer was formed during the hydrogen glow discharge cleaning before the main plasma experiments. Although the deposition of $\mathrm{Fe}$ and $\mathrm{Cr}$ would occur after the start of the main plasma experiment, the contributions of the main discharges to deposition layer formation are smaller than that of the hydrogen glow discharges.

\section{Discussions and Conclusion}

In LHD, which has carbon divertor plates, the reflectivity of the corner cube mirror is determined by the initial hydrogen glow discharge. The neon glow discharge and main plasma discharges do not seriously affect it. The reason for the significant degradation of the reflectivity during the hydrogen glow discharge was the deposition of carbon, which was released from the carbon divertor plate as hydrocarbon by chemical sputtering. Most of the deposition layer is formed during the initial hydrogen glow discharges. The reflectivity is decreased by the surface roughness, absorption by carbon, and interference between the multiple reflections in the deposition layer. The increase and decrease in the reflectivity could be attributed to the change in the thickness of the deposition layer, which changed the interference. The subsequent main and cleaning discharges have less effect on the deposition layer formation and reflectivity. It is not clear from this observation when the final interference pattern is formed. This is because the deposition particles tend to accumulate in the central region, as shown in Ref. [5]. It is possible that a uniform deposition layer (almost uniform color) on the mirror surface during the glow discharges might be sputtered to the central region from the peripheral region during the main discharges. Then, the slope of the deposition layer causes the interference pattern (gradual change in color). Closing the shutter during the hydrogen glow discharge, if possible, would be effective for maintaining the reflectivity, although the reliability of the mechanical components near the plasma should be confirmed.

On the other hand, the direct incoming of carbon from the divertor plates during the main discharges have to be noted. As reported in Ref. [9], the carbon seems to come from the specific direction, directly from the divertor plates, during the main discharges if the mirror faces the divertor. Because the mirror surface of the analysed corner cube mirror did not look to the nearest divertor plates as shown in Fig. 1, the deposition during the glow discharge would be dominant. It would be very rare that a corner cube mirror for laser diagnostics is installed near a divertor, and the mirror surface faces it. In this specific case, however, the carbon deposition during main discharges might increase, and additional measures, such as a cylinder with fins or a protective window [5], heating of the mirror, or in situ mirror cleanings, would be necessary. We speculated that the non-uniformity of the deposition on the three mirror surfaces might be explained as follows. Although the mirror surface of RM1 mainly faces the plasmas, the other two mirrors face the vacuum wall. There should be differences in the incoming impurities and charge exchange particles from plasmas among the three mirror parts. The difference in the mirror directions might cause the nonuniform deposition.

\section{Acknowledgments}

The authors thank Prof. Y. Hatano of Toyama University for surface analyses by GD-OES and fruitful discussion of the interpretation of the data. This study was supported by NIFS11KLEH009 and NIFS11ULHH027.

[1] D.L. Rudakov, J.A. Boedo, R.A. Moyer, A. Litnovsky et al., Rev. Sci. Instrum. 77, 10F126 (2006).

[2] V.S. Voitsenya, V.G. Konovalov, A.F. Shtan', S.I. Solodovchenko et al., Rev. Sci. Instrum. 70, 790 (1999).

[3] H. Iwakiri, K. Yasunaga, K. Morishita and N. Yoshida, J. Nucl. Mater. 283-287, 113 (2000).

[4] A. Litnovsky, P. Wienhold, V. Philipps, G. Sergienko et al., J. Nucl. Mater. 363-365, 1395 (2007).

[5] T. Akiyama, N. Yoshida, K. Kawahata, M. Tokitani et al., Nucl. Fusion 52, 063014 (2012).

[6] A. Litnovsky, V. Philipps, P. Wienhold, M. Matveeva et al., J. Nucl. Mater. 417, 830 (2011).

[7] Y. Zhou, L. Zhenga, Y.G. Lia, L.C. Lia et al., J. Nucl. Mater. 415, S1206 (2011).

[8] T. Akiyama, K. Kawahata, R. Yasuhara, S. Okajima and K. Nakayama, JINST 7, C01055 (2012).

[9] M. Tokitani, N. Yoshida, S. Masuzaki, N. Ashikawa et al., J. Nucl. Mater. 417, 668 (2011). 